\title{
A Neural Network Based Hybrid System for Detection, Characterization, and Classification of Short-Duration Oceanic Signals
}

\author{
Joydeep Ghosh, Larry M. Deuser, and Steven D. Beck
}

\begin{abstract}
Automated identification and classification of short-duration oceanic signals obtained from passive sonar is a complex problem because of the large variability in both temporal and spectral characteristics even in signals obtained from the same source. This paper presents the design and evaluation of a comprehensive classifier system for such signals. We first highlight the importance of selecting appropriate signal descriptors or feature vectors for high-quality classification of realistic short-duration oceanic signals. Wavelet-based feature extractors are shown to be superior to the more commonly used autoregressive coefficients and power spectral coefficients for this purpose. $A$ variety of static neural network classifiers are evaluated and compared favorably with traditional statistical techniques for signal classification. We concentrate on those networks that are able to time out irrelevant input features and are less susceptible to noisy inputs, and introduce two new neural-network based classifiers. Methods for combining the outputs of several classifiers to yield a more accurate labeling are proposed and evaluated based on the interpretation of network outputs as approximating posterior class probabilities. These methods lead to higher classification accuracy and also provide a mechanism for recognizing deviant signals and false alarms. Performance results are given for signals in the DARPA standard data set $I$.
\end{abstract}

Keywords-Neural networks, pattern classification, passive sonar, short-duration oceanic signals, feature extraction, evidence combination.

\section{INTRODUCTION}

$\mathrm{S}$ HORT-DURATION underwater signals obtained from passive sonar contain valuable clues for source identification in noisy and dissipative environments [1], [2]. Several biological sources such as sperm whale clicks, porpoise whistles, and snapping shrimps, as well as nonbiological phenomena such as ice crackles and mechanical sounds, produce characteristic sounds of a very short duration, typically 5 to $250 \mathrm{~ms}$. For example, porpoises radiate echolocation pulse trains, with each elementary pulse lasting from 15 to $20 \mathrm{~ms}$, and with an average of 40 ms between pulses. Such distinctive signatures can be

Manuscript received January 16, 1992, revised May 28, 1992. This work was supported by Grant N0001489-C-0298 with T. McKenna (ONR) and B. Yoon (DARPA) as Government cognizants.

J. Ghosh is with the Department of Electrical and Computer Engineering, University of Texas, Austin, TX 78712.

L. Deuser and S. Beck are with Tracor Applied Sciences, Austin, TX 78725

IEEE Log Number 9202281. identified by human experts either by ear or by looking at spectrograms of the processed sonar signals. However, attempts at automated classification of real-life acoustic signals based on purely spectral characteristics or on autoregressive modeling have met with very limited success over the past 25 years [3], [4].

A careful study of a large database of short-duration underwater signals available in-house at Tracor Applied Sciences, Inc., as well as the data sets provided by DARPA, shows several unique features of such signals that indicate why algorithm characterization and classification of oceanic acoustics is so difficult. These signals

- are highly nonstationary and impulsive;

- show significant variations in spectral characteristics and SNR due to differing sources or propagation paths, and due to multipath propagation;

- may overlap with one another;

- show rapid variation of spectral characteristics with both frequency and time; and

- often require event association for proper identification.

Some of these features can be observed in Fig. 1, which shows short-duration underwater signals due to a toadfish.

Artificial neural networks (ANN's) have several properties that make them promising for the automatic signal classification problem. They can serve as adaptive classifiers that learn through examples [5], [6]. Thus, they do not require a good a priori mathematical model for the underlying signal characteristics. This is advantageous since a comprehensive characterization of short-duration acoustic signals is not available yet. There are several neural networks that show comparable performance over a wide variety of classification problems, while providing a range of trade-offs in training time, coding complexity, and memory requirements [7], [8]. Some of these networks, including the multilayered perceptron when augmented with weight decay strategies [9], and the elliptical bias function network introduced in this paper, are quite insensitive to noise and to irrelevant inputs [10]. Moreover, a firmer theoretical understanding of the pattern recognition properties of feed-forward neural networks has emerged recently, that can relate their properties to 

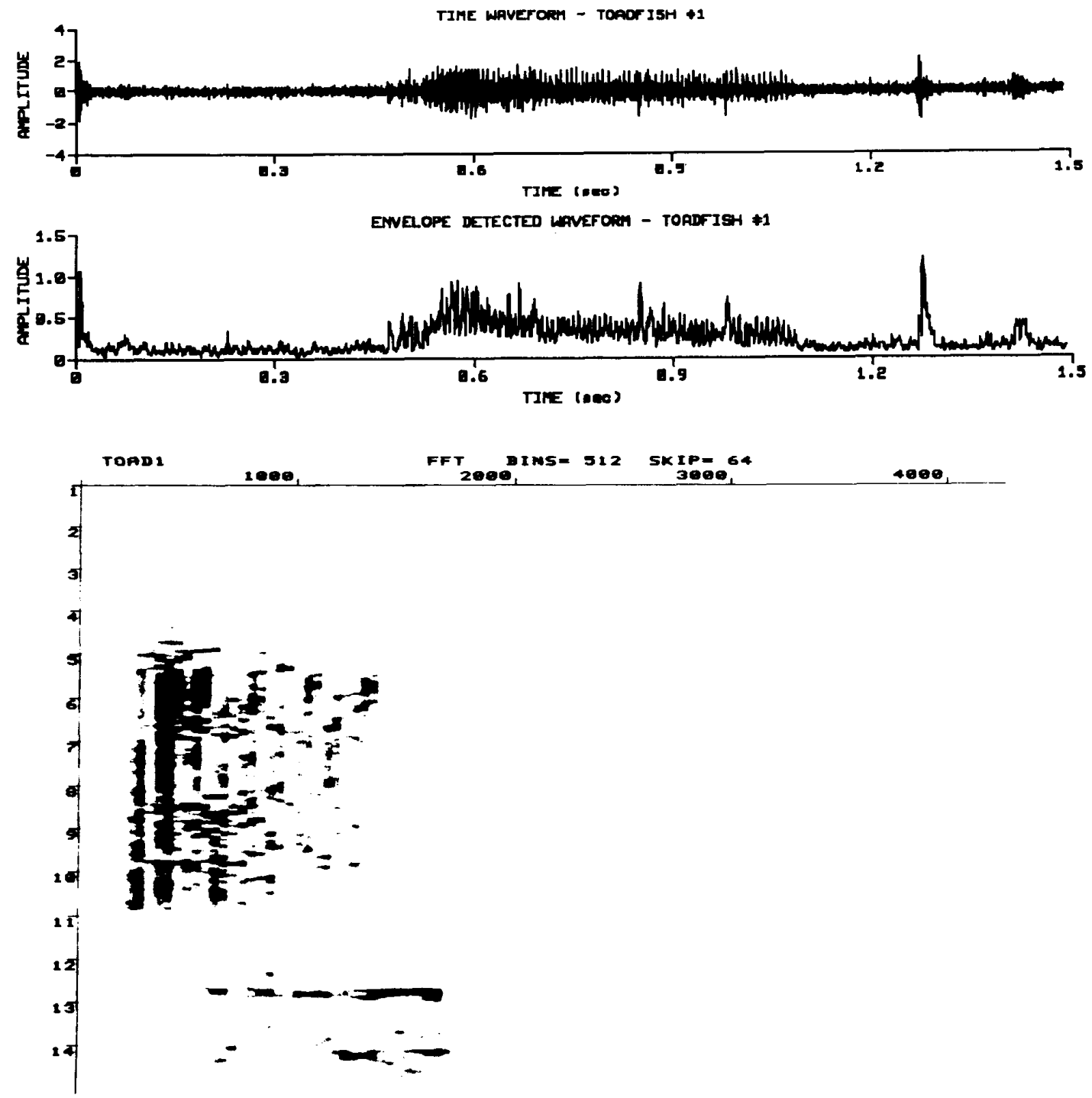

Fig. 1. Underwater acoustic signals due to a toadfish, displayed as time waveforms (above) and as a time-frequency spectrogram (below). The occurrence of a shorter duration signal (at around $t=1.3 \mathrm{~s}$ ) after the first signal of longer duration and lower frequency band, is characteristic of toadfishes. The association of these two events, together with some other signal features, enables one to distinguish toadfishes from other marine biological sources.

Bayesian decision making and to information theoretic results [11], [12].

Neural networks are not "magical." They do require that the set of examples used for training should come from the same (possibly unknown) distribution as the set used for resting the networks, in order to provide valid generalization and good performance on classifying unknown signals [13], [14]. Also, the number of training examples should be adequate and comparable to the number of effective parameters in the neural network, for valid results [12], [15], [16]. In this context, it is noted that cross-validation techniques can partially counter the effects of small training set size [12], [17].
This paper presents the design and evaluation of a comprehensive detection and classification system that uses a hybrid of ANN and statistical pattern recognition techniques tailored to recognizing short-duration oceanic signals [18]. Theoretical reasoning is provided for several of the design decisions, and performance results are given for the DARPA standard data set I. Fig. 2 shows the overall design of the hybrid classifier. Section II describes the preprocessing of the raw analog signals obtained from passive sonar and extraction of useful feature vectors from them. Our experience with alternative signal descriptors underscores the importance of selecting appropriate feature combinations in determining the classifica- 


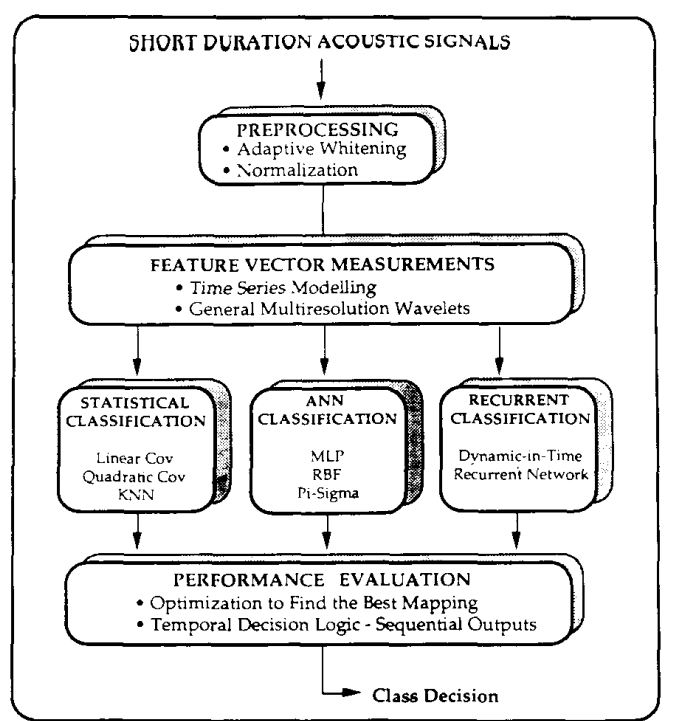

Fig. 2. Overall design of the signal detection and classification systems.

tion quality, irrespective of the classification techniques used [19]. The various types of ANN's used for classification are described in Section III, with particular emphasis on a novel local basis function classifier and the Pi-Sigma higher order network [20]. The DARPA standard data set I [21] is described in Section IV, and experimental results obtained by individual classifiers on this data set are presented. Section $\mathrm{V}$ introduces a high-level decisionmaking module which combines the evidences obtained from distinct classifiers for more accurate and robust results. Concluding remarks are given in Section VI.

\section{Signal Preprocessing and Feature EXTRACTION}

In order to classify events such as short-duration signals in a time series obtained from an underwater acoustic sensor, it is requisite that

i) all effects that vary, but not as a result of the events of interest, be removed or accounted for to the greatest possible extent; and

ii) the presence of each type of signal of interest should result in a measurable difference in the observable features.

These two requirements lead to the need for background normalization and feature extraction/selection respectively. One hallmark of our approach is that processing and classification is reformed on all input data without the usual signal-to-noise ratio (SNR) based prescreening to select signals for classification.

\subsection{Preprocessing}

The set of signal features that are extracted should be independent of the time-varying nose field and the sensor dynamics. Since the feature vectors are typically composed of combinations of broad-band and narrow-band energy estimates, the signal spectrum should be whitened across the entire band. Thus, the first step is to use an adaptive time-domain whitening filter to decorrelate the data from the long-time ambient noise, interference, and sensor characteristics, while passing short-duration signals relatively unchanged [22].

After a signal is extracted and pre-whitened, there are two basic choices for representing a signal in a form suitable as inputs to a neural network classifier.

i) A signal can be represented by a single vector that encodes some features of the signal. This vector is then used as an input for a static classifier such as those described in Section III. Previous works on sonar classification have most often used the normalized power spectra segmented into frequency bins (of equal or logarithmics size) to obtain the input vector. For example, input vectors based on the power spectra of passive sonar returns have been used in a multilayered perceptron network for a simple two-object problem [23], and also with a probabilistic neural network [24]. However, this simple approach is inadequate for more real-life shortduration oceanic signals. A vector obtained from the power spectra needs to be augmented by other temporal and spectral descriptors such as signal duration, peak frequencies, bandwidths, and possibly transformed, autoregressive (AR) model coefficients. Another technique is to use multiscale representations such as Gabor wavelets [25] to do not require assumptions of signal stationarity.

ii) Each signal can be represented by a series of feature vectors sequenced in time [26]. Each feature vector is a descriptor of the signal observed within a particular time window. A set of overlapping time windows is used to obtain the sequence. For example, a sequence of vectors obtained by the energy in discrete frequency bins extracted through successive time windows over the input signal, can be used to form such a composite vector. Note that such representations explicitly recognize the inputs to be spatiotemporal signals, and are suited for dynamic classifiers mentioned in Section 3.1. The set of features extracted from a signal can also be regarded as a two-dimensional input for use in models such as time-delay neural networks. [6].

\subsection{Feature Vector Selection}

It was observed in our previous study [19], [21] that discriminant parameters obtained using wavelet transforms yield better performance than those using AR modeling or spectral coefficients. We note that both AR modeling and cepstral coefficients are sensitive to the SNR of the signal, the phase, and the modeling order. Many oceanic signals are embedded in significant noise, mostly broad-band. Phase depends on the estimated starting point of the signal, which is difficult to determine even with a good detector. Setting a high SNR threshold for 
lower false alarm also results in a poorer phase estimate. Finally, both broad-band and narrow-band signals are important, and too high an order results in noisy coefficients.

To extract the features, a constant-Q prototype wavelet with 24 coefficients is used, in addition to signal time duration to characterize the signals in our current work. Constant-Q analyzing function is broad-band and of very short duration at the higher frequencies, corresponding to better SNR representation of impulsive sounds. At lower frequencies, the bandwidth is narrower and the duration longer.

A good overview of wavelet transforms can be found in [27]. The particular wavelet transformer used in this paper represents a signal $x(t)$ by shifted and dilated version of an analyzing waveform [25]:

$$
T_{x}(\tau, a)=a^{-m / 2} \int_{-\infty}^{\infty} x(t) g^{*}\left(\frac{t-\tau}{a^{m}}\right) d t
$$

where the phototype wavelet is given by

$$
g(t)=a^{-m / 2} g\left(\frac{t-\tau}{a^{m}}\right) d t .
$$

Here $a$ is a scaling factor, $m$ the scaling index, and * indicates the complex conjugate. The choice of the most appropriate prototype function is still an open research issue. To date, fractional octave frequency spacing has proved quite successful.

Another important classification parameter is the signal duration. In fact, in the DARPA Standard Data Set I, signal duration is the only discriminant between classes $C$ and $\mathrm{D}$. The duration of realistic acoustic signatures can vary over two orders of magnitude. The importance of this metric is underscored by the recent work by French researchers [28] who have used a two-stage architecture for classifying oceanic signals. The first stage extracts the signals and sorts them into different categories according to signal duration. The second stage then uses a neural classifier for each category. Unfortunately, this approach is computationally expensive when there is a wide range of signal durations, and is also limited in quality of results. In the experiments reported in this paper, time duration is simply used as an added classification parameter. Thus the actual dimension of each feature vector is 25 (24 wavelet coefficients plus time duration) when waveletbased features are used.

Irrespective of the approach taken, the use of large, possibly composite, feature vectors can become computationally expensive. If the resultant input is of high dimension, it forces us to use a network with a higher number of free or effective parameters. This leads to a classical estimation problem if the training set size is small in comparison, wherein the presence of a larger number of parameters can result in a solution that is over-determined [29]. Also, high-dimensional feature vectors are more susceptible to noise. Moreover, the presence of irrelevant feature combinations can actually obscure the impact of the more discriminating inputs.

An obvious solution to these problems is to reduce each feature vector to a much lower dimensional vector for actual presentation to the classification networks. We tried this approach by extracting the most significant principal components using Sanger's method [30], and also implemented a modified version of Kohonen's selforganizing feature maps described in [31] as an alternate technique for dimensionality reduction. Both these approaches did not fare well due to the nonstationary nature of the signal and the small size of the training set. This led us to tackle the problem with the classification networks themselves, by using variations of ANN classifiers that are more effective for high-dimensional inputs and more robust against noise. This issue is further discussed in Section III.

\section{Neural Network Classifiers}

ANN approaches to problems in the field of pattern recognition and signal processing have led to the development of various "neural" classifiers using feed-forward networks [32], [33]. These include the multilayer perceptron (MLP) as well as kernel-based classifiers such as those employing radial basis functions (RBF's) [34], [35]. A second group of neural-like schemes such as learning vector quantization (LVQ) have also received considerable attention [31]. These are adaptive, exemplar-based classifiers that are closer in spirit to the classical $K$-nearest neighbor method.

The strength of both groups of classifiers lies in their applicability to problems involving arbitrary distributions. Most neural network classifiers do not require simultaneous availability of all training data and frequently yield error rates comparable to Bayesian methods without needing a priori information. Techniques such as fuzzy logic can be incorporated into a neural network classifier for applications with little training data [36]. A good review of probabilistic, hyperplane, kernel and exemplarbased classifiers that discusses the relative merit of various schemes within each category, is available in [32], [33]. Comparisons between these classifiers and conventional techniques such as decision trees, $K$-nearest neighbor, Gaussian mixtures, and CART can be found in [33] and [38].

\subsection{Static versus Dynamic Approaches}

For all of the classifiers mentioned above, each signal needs to be represented by a single feature vector rather than as a spatiotemporal pattern that changes with time. This is why such classifiers are often referred to as "static" systems [32]. Indeed, almost all of the neural network classifiers that have been studied and used so far fall into this category. Since static classifiers are based on reduced input representations, they have an inherent drawback in that information contained in the temporal variations in the signal may not get recorded. Since many shortduration oceanic signals such as whale cries, have characteristics such as FM slides, important discriminatory evidence may be lost when each signal is represented by a single vector. This motivates the use of dynamic classifiers 
that base their decisions on a sequence of feature vectors corresponding to different, possibly overlapping, time intervals. The appropriate signal representation for such dynamic classifiers corresponds to the second choice mentioned in Section II.

A dynamic neural classifier can be implemented through recurrent networks that can store past history feedback connections among the processing cells. These networks can be used to classify waveforms of arbitrary duration using a network of fixed complexity, and have been used successfully in speech recognition [6]. Dynamic recurrent networks are of three main types.

a) Partial recurrent networks using context units involve feedback to selective cells in order to record temporal sequences. Notable examples are networks that use feedback from output units to the input layer [39] or specify a context from hidden cells [40]. We observe that units with feedback connections accumulate an exponentially decaying weighted sum of current and past values to construct a static representation of a temporal input sequence. Such an architecture avoids two deficiencies found in other models of sequence recognition: first, it reduces the difficulty of temporal credit assignment by focusing the backpropagated error signal; second, it eliminates the need for a buffer to hold the input sequence and/or intermediate activity levels. However, they require clocked inputs, and are susceptible to spatio temporal warping [41].

b) Real-time recurrent backpropagation and timedependent recurrent backpropagation [42] do not require clocked inputs, but are very sensitive to learning rates.

c) Dynamic-in-time networks that continuously update confidences of the input belonging to each class as feature vectors are presented in sequence, and makes a decision if the confidence factor exceeds a threshold for some class. We are currently investigating one such model for classifying oceanic signals.

Overall, dynamic classifiers are more powerful and promising for complex temporal patterns. At present, the candidate dynamic classifiers are very susceptible to signal misalignment or registration problems and to spatiotemporal warping. They also need longer training schedules and easily exhibit divergent behavior unless the adaptation parameters are fine-tuned and the inputs have little noise components. For these reasons, we do not consider dynamic classifiers any further in this paper, while recognizing their potential.

\subsection{Design Considerations and Trade-offs}

Besides the MLP, RBF, and LVQ type static classifiers mentioned above, there are several other neural-like candidates such as higher order polynomial GMDH classifiers and functional-link networks [43], [44]. Given such a wide variety, what should be the criteria for determining the most suitable ones for classifying short-duration oceanic signals? Our experiences, corroborated by those of several other researchers (see [33], for example), show that classification error rates are similar across different classifiers when they are powerful enough to form minimum error decision regions, when they are properly tuned, and when sufficient training data is available. Practical characteristics such as training time, classification time, and memory requirements, however, can differ by orders of magnitude. Also, the classifiers differ in their robustness against noise, effects of small training sets, and in their ability to handle high-dimensional inputs [10]. These factors, rather than small differences in the best possible error rates, should form the basis of our network selection process.

RBF networks are primarily aimed at multivariate function interpolation or function approximation, and have been used successfully for problems such as prediction of chaotic time series [35]. They serve as universal approximators using only a single hidden layer [45]. However. they can also be used for classification. For example, Niranjan and Fallside were able to achieve good results on voice and digit speech categorization by using one "centroid" for each training vector [46]. The results are robust with respect to variations in the class distributions. Thus, our first candidate for a short-duration oceanic signal classifier is an adaptive, kernel-based network described in Section 3.3.

We observe that $L V Q$ and it variants such as LVQ2, LVQ2.1, and "conscience learning" [47] need somewhat less training time than an MLP-based classifier for comparable performance [37]. The memory requirements are similar but their performance is more sensitive to initial choice of reference vectors. Networks using RBF's, on the other hand, need much shorter training times at the expense of additional memory as compared to the MLP. The localized response of hidden units in kernel-based networks such as the RBF network, as compared to the global responses of MLP networks, make them suitable for detecting atypical signals or false alarms since they result in low values of network outputs. This motivates us to investigate hybrid networks that combine the best features of LVQ and RBF based classifiers so that an accurate classifier is obtained that requires less training time and is not memory intensive. A novel hybrid network that achieves these objectives is also discussed in Section 3.3, and forms our second classifier candidate.

Higher order networks based on the GMDH algorithm often require long training times as well as large amount of memory to yield comparable error rates [8]. Polynomial networks based on Volterra series expansion [48], [49] show fairly stable, single-layer learning, but the number of weights involved grows exponentially with the order of the network. We have recently proposed a higher order network called the Pi-Sigma network, which is able to maintain the capabilities of polynomial networks while greatly reducing network complexity [20], [50]. It is also able to incrementally grow until a desired level of complexity is 
reached. This network is the third classifier candidate, and is described briefly in Section 3.4.

MLP networks that adapt weights using gradient descent of the mean squared error (MSE) in weight space, are perhaps the most commonly used neural network classifiers. These networks are capable of approximating any arbitrary bounded measurable function defined over a compact set, given sufficient number of hidden units [51]. The generalization capability of these networks can be enhanced by restricting the weight-space through adding extra terms to the cost functions or by selective pruning of weights [9], [13]. Weight pruning techniques also serve to reduce the effective number of parameters [15], making the resultant "parsimonious" feedforward networks more tailored to noisy high-dimensional inputs. Moreover, the first hidden layer of an MLP can be considered as feature extractors especially if localized connections are used.

Countering the advantages of an MLP mentioned above, are the problems of high training times, and of choosing an appropriate network size. As noted by Makhoul [12], "the sigmoid, because of its sharp transition... focuses attention during training on data samples that are confusable. Basically, it helps specify the boundary between the samples within and outside the class. Thus, in general, much of the training data does not participate in determining the network parameter values." We note that this drawback is even more severe when the number of training samples is limited. For all those reasons, the MLP is not considered further in this paper, and instead the reader is referred to [52] for our results on using an MLP with optimal brain damage [9] for classifying underwater signals from biologic sources.

\subsection{Efficient Adaptive Kermel Classifiers}

We first summarize the LVQ and RBF procedures and then introduce two hybrid networks that attempt to incorporate the best of both LVQ and RBF techniques.

LVQ is an adaptive version of the classical vector quantization algorithm whose aim is to represent a set of vectors by a smaller set of codebook vectors and reference vectors (RV's) so as to minimize an error functional. Typically, the algorithm consists of the following steps: The RV's are initialized by a random selection or from $K$-means clustering on the training set. These vectors are now adjusted iteratively by moving them closer to or further away from training inputs depending on whether the closest RV is of the same class as the input vector or not. Unknown inputs are assigned the class of the nearest $\mathrm{RV}$, with the Euclidean norm being the most common measure of distance. Let the $n$th training pattern vector $\boldsymbol{x}(n)$ belong to class $C_{r}$, and $\boldsymbol{m}_{\boldsymbol{c}}(n)$, the reference vector closest to $\boldsymbol{x}(n)$, belong to class $C_{s}$. At the presentation of the $n$th input, the RV's are adapted as follows.

$$
\begin{aligned}
\boldsymbol{m}_{c}(n+1) & =\boldsymbol{m}_{c}(n)+\alpha(n)\left[\boldsymbol{x}(n)-\boldsymbol{m}_{c}(n)\right] \\
\boldsymbol{m}_{c}(n+1) & =\boldsymbol{m}_{c}(n)-\alpha(n)\left[\boldsymbol{x}(n)-\boldsymbol{m}_{c}(n)\right] \\
\text { if } C_{r} & \neq C_{s} \\
\boldsymbol{m}_{i}(n+1) & =\boldsymbol{m}_{i}(n) \quad \text { if } C_{r}=C_{s}
\end{aligned}
$$

where $\alpha(n)$ is a leaning factor that decreases monotonically with time. Detail of LVQ-type learning procedures can be found in [31], [47], and [53].

$R B F$ networks are a class of signal hidden-layer feedforward networks in which radially symmetric basis functions are used as the activation functions for the hidden layer units. A generic RBF network is shown in Fig. 3. Let $x_{p}=\left(x_{p 1}, x_{p 2}, \cdots, x_{p N}\right)^{T}$ denote the $p$ th $N$-dimensional input. When this input is presented to the RBF network, the output of the $j$ th hidden node, $R_{j}\left(x_{p}\right)$, and that of the $i$ th output node, $f_{i}\left(x_{p}\right)$, are given by

$$
\begin{gathered}
f_{i}\left(\boldsymbol{x}_{\boldsymbol{p}}\right)=\sum_{j} w_{i j} R_{j}\left(\boldsymbol{x}_{\boldsymbol{p}}\right) \\
R_{j}\left(\boldsymbol{x}_{\boldsymbol{p}}\right)=R\left(\frac{\left\|\boldsymbol{x}_{\boldsymbol{p}}-\boldsymbol{x}_{j}\right\|}{\sigma_{j}}\right)
\end{gathered}
$$

where $R(\cdot)$ is a radially symmetric function such as a Gaussian. In the above, $x_{j}$ is the location of the $j$ th centroid, where each centroid is a kernel/hidden node, $\sigma_{j}$ is a scalar denoting the "width" of its receptive field and $w_{i j}$ is the weight connecting the $j$ th kernel/hidden node to the $i$ th output node. For Gaussian RBF's the width $\sigma_{j}$ is the standard deviation, so that we have

$$
R_{j}\left(x_{p}\right)=e^{-1 / 2\left(\left\|x_{p}-x_{j}\right\|^{2} / \sigma^{2}\right)} .
$$

Hybrid Kernel Classifiers: Both LVQ and RBF involve construction of a representative set of the training data-the centroids or hidden units of RBF and there reference vectors of $L V Q$ - which determine the final decision. Previously, the controids in an RBF network were determined using heuristics such as performing $k$ means clustering on the input set, and widths were held fixed during training. Alternatively, we can vary both the centroid locations and associated widths of the receptive fields by performing gradient descent on the mean square error of the output. This leads to the adaptive kernel classifier (AKC).

Adaptive Kernel Classifier: Consider a quadratic error function, $E=\sum_{p} E_{p}$, where $E_{p}=1 / 2 \sum_{i}\left(t_{i}^{p}-f_{i}\left(\boldsymbol{x}_{p}\right)\right)^{2}$. Here $t_{i}^{p}$ is the $i$ th component of the target function for input $\boldsymbol{x p}$, and $f_{i}\left(\boldsymbol{x}_{\boldsymbol{p}}\right)$ is the corresponding network output as defined in (4). The mean square error is the expected value of $E_{p}$ over all patterns. Let $\Delta w_{i j}, \Delta x_{j k}$, and $\Delta \sigma_{j}$ represent the change in weight $w_{i j}$, location of $k$ th component of the $j$ th centroid, and the width, $\sigma_{j}$ of this centroid respectively, at each learning step. The update rules for these network parameters are obtained using gradient descent on $E_{p}$, and are given by

$$
\begin{aligned}
& \Delta w_{i j}=\eta_{1}\left(t_{i}^{p}-f_{i}\left(x_{p}\right)\right) R_{j}\left(x_{p}\right) \\
& \Delta x_{j k}=\eta_{2} R_{j}\left(x_{p}\right) \frac{\left(x_{p k}-x_{j k}\right)}{\sigma_{j}^{2}}\left(\sum_{i}\left(t_{i}^{p}-f_{i}\left(x_{p}\right)\right) w_{i j}\right) \\
& \Delta \sigma_{j}=\eta_{3} R_{j}\left(x_{p}\right) \frac{\left\|x_{p}-x_{j}\right\|^{2}}{\sigma_{j}^{3}}\left(\sum_{i}\left(t_{i}^{p}-f_{i}\left(x_{p}\right)\right) w_{i j}\right) .
\end{aligned}
$$




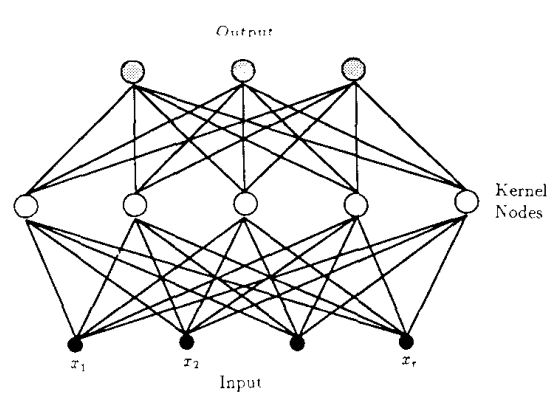

Fig. 3. An RBF network.

These equations constitute the learning scheme for the AKC. A similar scheme, called the Gaussian potential function network (GPFN), which involves segmentation of the input domain into several potential fields in the form of Gaussians, was proposed by Lee and Kil [54]. The Gaussian potential functions of this scheme need not be radially symmetric functions. Instead, the sizes of these potential fields are determined by a correlation matrix. The network parameters are computed by gradient descent as in the case of $\mathrm{AKC}$. We studied a network of nonradial basis functions with a different smoothing factor in each dimension. Thus, (4) is used with

$$
R_{i}\left(x_{p}\right)=e^{1 / 2 \sum_{k}\left(\left(x_{p \cdot k}-x_{j k}\right)^{2} / \sigma_{j, \frac{1}{k}}\right)}
$$

and, in general $\sigma_{j k} \neq \sigma_{i j}$. For this case update rules (7) and $(8)$ become

$$
\begin{aligned}
& \Delta x_{j k}=\eta_{2} R_{j}\left(x_{p}\right) \frac{\left(x_{p k}-x_{j k}\right)}{\sigma_{j k}^{2}}\left(\sum_{i}\left(t_{i}^{p}-f_{i}\left(x_{p}\right)\right) w_{i j}\right) \\
& \Delta \sigma_{j k}=\eta_{3} R_{j}\left(x_{p}\right) \frac{\left(x_{p k}-x_{j k}\right)^{2}}{\sigma_{j k}^{3}}\left(\sum_{i}\left(t_{i}^{p}-f_{i}\left(x_{p}\right)\right) w_{i j}\right) .
\end{aligned}
$$

Classification experiments show that these elliptical basis function networks require considerably shorter training time compared to the $\mathrm{AKC}$ and typically require a fewer number of kernel nodes. To further speedup network training, we suggest replacement of the parameter $\sigma$ with a new parameter $\alpha$ by making the substitution $\sigma=$ $1 / \alpha$. This eliminates all division operations involved in training and testing, which are known to be computationally expensive.

How should the widths, $\sigma_{i k} s$ be initialized? For RBF, the initial positions of the centroids are typically obtained by $k$-means clustering, and the width $\sigma_{j}$ for the $j$ th centroid is of the same order as the distance between this unit and the nearest centroid, $x_{j}$. This suggests the initialization

$$
\sigma_{j k}(\text { init })=\sigma \times\left\|x_{j}-x_{j^{*}}\right\|, \quad \forall j, k .
$$

However, since the spread of data is in general different in different dimensions, an initialization given by

$$
\sigma_{j k}(\text { init })=n^{1 / 2} \sigma \times\left\|\boldsymbol{x}_{j k}-\boldsymbol{x}_{\boldsymbol{j} * k}\right\|, \quad \forall j, k
$$

seems more appropriate, where $\sigma=O(1)$ determines selectivity, and $n^{1 / 2}$ is a normalization term for $n$-dimensional inputs so that the average variance is $\sigma_{i}^{2}$, as before.

More general schemes like the regularization networks have been studied by Poggio et al. [55]. Though more complex decision regions can be shaped out of potential fields that are not radially symmetric, receptive fields of radial functions can achieve universal approximation even if each kernel node has the same smoothing factor, $\sigma$ [56]. The principal advantage of the AKC is that it is able to perform the same level of approximation as RBF using fewer hidden units. However, training time is increased since centroids and center widths are also adapted using the generalized delta rule, which is a slower procedure.

Rapid Kemel Classifier: Hybrid schemes that combine unsupervised and supervised learned in a single network have been proposed in [5] and [57]. For instance, the hierarchical feature map classifier of Huang and Lippmann [5] consists of a feature map stage followed by a stage of adaptive weights. The central idea behind these approaches is to have a layer that is trained in an unsupervised way followed by a layer that will be trained using the delta rule. Since backpropagation of error through multiple layers is avoided these hybrid networks yield remarkable speedups in computation. Such methods are particularly useful when there are a large number of training samples. It must be noted that these hybrid schemes are not optimized in the manner of backpropagation since the first stage parameters are not optimized with respect to the output performance. A question that immediately arises is: how does the location of centroids obtained by hybrid training compare with that obtained by strict gradient descent?

Keeping in view the similarities on form between the equations describing update of centroids by the delta rule and the LVQ algorithm, one can replace the former equation with the latter in the AKC training scheme. This results in the rapid kernel classifier (RKC) shown in Fig. 4 , which requires shorter training times with little change in performance as compared to the AKC. In the hybrid procedures mentioned above various layers of the network are trained sequentially. The first stage parameters are first trained in an unsupervised way and are held fixed during the second stage training. On the contrary, in the RKC scheme we let the LVQ algorithm run in parallel with the training of the second layer. We note that a variant, RKCEB, can also be studied in which elliptical basis functions are used.

Interestingly, in all our experiments so far with $\mathrm{RKC}$, the mean square error decreases monotonically as in the case when all the parameters are adapted by backpropagation. This indicates that adjusting the centroids using 


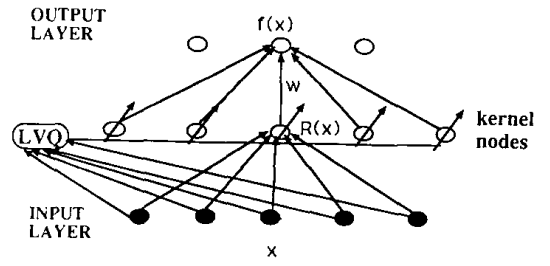

Fig. 4. An RKC.

LVQ might amount to performing gradient descent on the "centroid-location space." While proving this seems difficult, we have been able to obtain preliminary results indicating such a connection [58].

The distinguishing features of the various localized networks introduced in this section are summarized in Table I.

\subsection{Higher Order Pi-Sigma Networks}

The recently introduced pi-sigma networks (PSN's) [20], [50] are higher order networks whose name stems from the fact that these networks use product of sums of input components instead of sums of products as in "sigma-pi" units, to obtain the outputs. The primary motivation for these networks is to develop a systematic method for maintaining the fast learning property and powerful mapping capability of single layer higher-order networks while avoiding the combinatorial increase in the number of weights and processing units required.

Fig. 5 shows a PSN with a single output. This network is a fully connected two-layered feedforward network. However, the summing layer is not "hidden" as in the case of the multilayered perceptron (MLP), since weights from this layer to the outputs are fixed at 1 . This property drastically reduces training time.

Let $\boldsymbol{x}=\left(1, x_{1}, \cdots, x_{N}\right)^{T}$ be an $N+1$-dimensional augmented input column vector where $x_{k}$ denotes the $k$ th component of $\boldsymbol{x}$. The inputs are weighted by $k$ weight vectors $\boldsymbol{w}_{j}=\left(w_{0 j}, w_{1 j}, \cdots, w_{N j}\right)^{T}, j=1,2, \cdots, K$ and summed by a layer of $K$ linear "summing" units, where $K$ is the desired order of the network.

The output of the $j$ th summing unit, $h_{j}$, is given by

$$
h_{j}=\sum_{k=1}^{N} w_{k j} x_{k}+w_{0 j}, \quad j=1,2, \cdots, K .
$$

The output $y$ is given by

$$
y=f\left(\prod_{j=1}^{K} h_{j}\right)
$$

where $f(\cdot)$ is a suitable nonlinear activation function, and is chosen as the sigmoid function:

$$
f(x)=\frac{1}{1+e^{-x}}
$$

for our purposes. In (12) and (13), $w_{k j}$ is an adjustable weight from input $x_{k}$ to $j$ th summing unit and $w_{0 j}$ is the

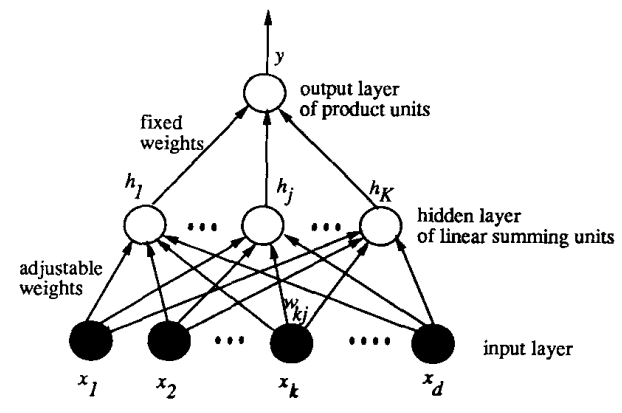

Fig. 5. A pi-sigma network with one output.

TABLE I

\begin{tabular}{lllr}
\hline & \multicolumn{3}{c}{ Adaptation of Network Parameters } \\
\cline { 2 - 4 } Type & \multicolumn{1}{c}{ Centroid } & Weights & \multicolumn{1}{c}{ Widths } \\
\hline RBF & K-means / fixed & Delta rule & Fixed; isotropic \\
AKC & Delta rule & Delta rule & Delta rule; isotropic \\
EBF & Delta rule & Delta rule & Delta rule; anisotropic \\
\hline RKC & LVQ & Delta rule & Delta rule; isotropic \\
RKCEB & LVQ & Delta rule & Delta rule; anisotropic \\
\hline
\end{tabular}

threshold of the $j$ th summing unit. The weights can take arbitrary real values. If a specific input, say, $\boldsymbol{x}_{\boldsymbol{p}}$ is considered, then the $\left(h_{j}\right)$ 's, $y$, and net are superscripted by $p$.

The network shown in Fig. 5 is called a $K$ th-order PSN since $K$ summing units are incorporated. The total number of adjustable weight connections for a $k$ th order PSN with $N$ dimensional inputs is $(N+1) \cdot K$. If multiple outputs are required, an independent summing layer is needed for each output. Thus, for an $M$-dimensional output vector $y$, a total of $\sum_{i=1}^{M}(N+1) \cdot K_{i}$ adjustable weight connections are needed, where $K_{i}$ is the number of summing units for the $i$ th output. This allows us great flexibility since all outputs do not have to retain the same complexity. Note that using product units in the output layer indirectly incorporates the capabilities of higher order networks with a smaller number of weights and processing units. This also enables the network to be regular and incrementally expandable, since the order can be increased by one by adding another summing unit and associated weights, but without disturbing any connection established previously.

An asynchronous adaptation rule is used in which only those weights corresponding to the $l$ th summing, randomly chosen for each input, is updated per input sample. Such a rule leads to more stable learning than updating all weights at each step. Gradient descent on the estimated MSE leads to the following update rule:

$$
\Delta \boldsymbol{w}_{l}=\eta \cdot\left(t^{p}-y^{p}\right) \cdot\left(y^{p}\right)^{\prime} \cdot\left(\prod_{j \neq l} h_{j}^{p}\right) \cdot \boldsymbol{x}_{\boldsymbol{p}}
$$

where $\left(y^{p}\right)^{\prime}$ is the first derivative of sigmoidal function $\sigma(\cdot), x_{p}$ is the (augmented) $p$ th input pattern, and $\eta$ is the learning rate.

PSN's have been successfully applied to several prob- 
lems involving function approximation or pattern classification. A second- or third-order network is usually sufficient for obtaining a reasonably good decision surface in very short time. Another advantage is that more difficult signals can use a network with more summing units, without disturbing the smoother lower order generalization for simpler signal classes. The behavior of PSN's is well characterized mathematically, and bounds on the learning rate, $\eta$ for convergence, can be found in [50].

\subsection{Statistical Classifiers}

Besides the neural network classifiers, we also considered several traditional classifiers based on the theory of statistical pattern recognition [59]. [60]. The first is the $K$-nearest neighbor (KNN) classifier, which is a kernelbased technique. The KNN assigns to an unclassified data sample the identity of the majority of the nearest (in a Euclidean sense) $K$ preclassified samples from the training data. While the error rate is typically greater than the Bayes optimal risk, the KNN error is bounded by twice the Bayes optimal risk. Since our number of training samples per class ranged from 5 to $9, K=3$ was chosen. Note that since every training sample has to be considered in the labeling process, the memory and computational requirements increase linearly with the training data size. Also, this technique cannot differentiate among simultaneously occurring signals since it does not decompose an input vector.

The second classifier considered is the generalized Fisher linear discriminant, often referred to as the linear classifier (LC). The LC uses the lumped covariance matrix, $R$, and the individual class dependent means, $M_{i}$, to compute

$$
V(j, X)=\left(X-M_{j}\right)^{T} R{ }^{\prime}\left(X-M_{j}\right)
$$

for each class, and declares the membership of input $X$ to be same as that $j$ for which the value of $V(j, X)$ is the least. For the shot duration signals in the DARPA Standard Data Set I, singularities in $R$ were found. These were handled using singular value decomposition techniques to perform the matrix inversion and a step-wise regression to determine good features. Although the LC is easy to use, it is cumbersome to update, and degrades rapidly as the Bayes optimal decision boundaries become highly nonlinear. The performance of the $\mathrm{LC}$ is given in [21], and is not repeated here because it is inferior to all the other techniques considered. Similarly, the results of using a quadratic classifier are omitted since they were much more computationally intensive without showing a significant improvement in classification accuracy as compared to the LC.

\section{PERformance EVAiuntion}

\subsection{Data Description and Representation}

The various classifiers described in the previous section have been evaluated for their ability to classify short duration acoustic signals extracted from DARPA Stan- dard Data Set I [18]. This data set consists of digital time records of a training and a test set of six signal types propagated over short oceanic data paths with variable source and background noise levels. For the results reported in this paper, only those portions of the records that contain signals are used. As shown in Table II, the six signal classes are denoted by the letters $A$ through $F$ with durations from a few milliseconds to $8 \mathrm{~s}$, and bandwidths from less than $1 \mathrm{~Hz}$ to several $\mathrm{kHz}$. The SNR's of the records vary over $24 \mathrm{~dB}$. There were 42 training samples. Of the test data, 179 samples were similar enough to the training data to constitute a good test set for classification comparisons. An additional 19 test samples were added selectively to observe, without retraining, the robustness of the techniques to extraneous deterministic signals. These 19 additional records had duration and frequency characteristics that deviated significantly from the training data, and are thus identified by primed labels in Table II. Overall, the data set included several signal characteristics that are representative of realistic oceanic signals, including

i) Simultaneously occurring signals (some type $F$ signals, several type $B$ signals).

ii) Sequentially occurring signals requiring event association.

iii) Signals similar to type $C$ or type $A$ but with some important feature missing.

iv) Significant signal variations due to the source or propagation path; possibly low SNR.

From the size of the training set, it is apparent that the training data is small compared to the number of free parameters for all the neural networks considered, with the problem being most severe for the basis function networks and least for the PSN. This sparse data problem is dealt with by using cross-validation, wherein part of the training data is also used to test the network at every training cycle, and training is stopped when the performance reaches a peak and begins to fall. In this way, the network is encouraged not to memorize the training data, and can do better generalization when faced with unknown data.

\subsection{Performance of Individual Classifiers}

The adaptive and rapid kernel classifiers were compared with the LVQ2 and RBF, as well as the KNN classifier and the Pi-Sigma higher order network [20] in terms of classification accuracy, training and test times, and memory requirements. The LVQ2 and the two hybrid algorithms used five reference vectors for each class, while the RBF used a total of 15 centroids. For the KNN, $K=3$ was chosen, and the number of training samples ranged between 5 and 9 per class for total of 42 samples. The PSN used three hidden units, and was trained using all 42 samples. The number of iterations, determined by a cut-off in differential MSE of 0.001 was 35,104 and 816 for the RKC, AKC, and PSN's, respectively. 

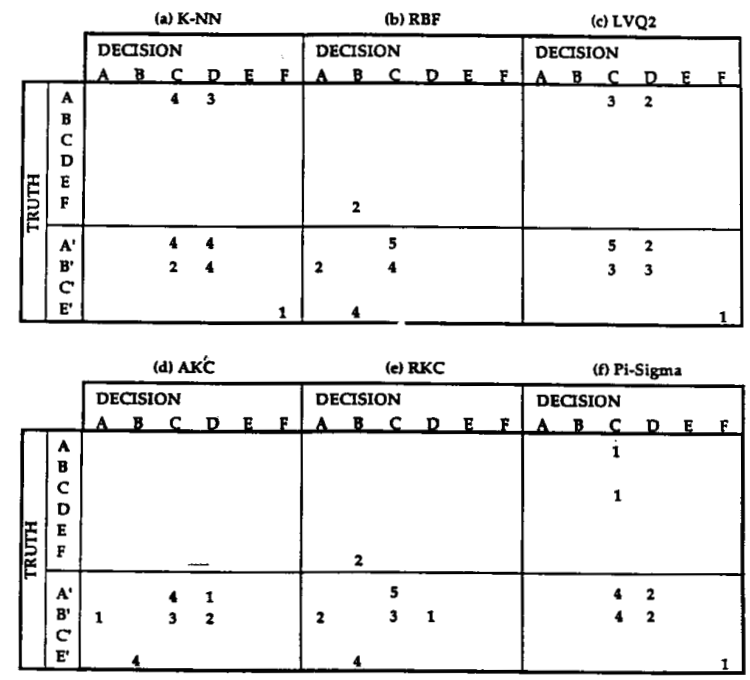

Fig. 6. Confusion matrices showing classification error results for (a) 3-nearest neighbor, (b) RBF, (c) LVQ2, (d) adaptive kernel classifier, (e) rapid kernel classifier, (f) pi-sigma network.

TABLE II

\begin{tabular}{|c|c|c|c|c|}
\hline \multicolumn{5}{|c|}{ Description of DARPA Standard Data Set I } \\
\hline \multicolumn{2}{|r|}{ Signal Classes } & \multicolumn{3}{|c|}{ Number of Samples } \\
\hline Class & Description & Training & Test 179 & $\begin{array}{l}\text { Additional } \\
19 \text { Deviant } \\
\text { Test Signals }\end{array}$ \\
\hline A & Broad-band $15 \mathrm{~ms}$ pulse & 7 & 53 & $8\left(A^{\prime}\right)$ \\
\hline B & Two $4 \mathrm{~ms}$ pulses, $27 \mathrm{~ms}$ separation & 7 & 54 & $6\left(B^{\prime}\right)$ \\
\hline $\mathrm{C}$ & $3 \mathrm{kHz}$ tonal, $10 \mathrm{~ms}$ duration & 8 & 31 & $1\left(C^{\prime}\right)$ \\
\hline $\mathrm{D}$ & $3 \mathrm{kHz}$ tonal, $100 \mathrm{~ms}$ duration & 9 & 14 & $0\left(D^{\prime}\right)$ \\
\hline $\mathrm{E}$ & $150 \mathrm{~Hz}$ tonal, $1 \mathrm{~s}$ duration & 6 & 19 & $4\left(E^{\prime}\right)$ \\
\hline $\mathrm{F}$ & $250 \mathrm{~Hz}$ tonal, $8 \mathrm{~s}$ duration & 5 & 8 & $0\left(F^{\prime}\right)$ \\
\hline & Totals & 42 & 179 & 19 \\
\hline
\end{tabular}

The classification results for the six different techniques, examined only for the signal epochs, are displayed in Fig. 6 using confusion matrices. For each technique, the correct class is given by the horizontal row label and the misclassification (or confused) class assigned is given by the vertical column label. The numerical entries give the number of misclassifications. Such a display format exposes which signals cause the greatest problems for a given technique. The upper half of the matrix shows the results for the 179 test signals, while the lower half, identified by the primed labels $A^{\prime}$ through $E^{\prime}$, display results for the 19 deviant test signals. We note that the $\mathrm{AKC}$ is able to classify all regular test signals correctly $(100 \%)$ while the $3-\mathrm{NN}$ gave the poorest results $(7 \mathrm{mis}-$ classifications or $96.1 \%$ accuracy). For the deviant signals, all six techniques could label only 4 to $6(21.1 \%-31.6 \%)$ in agreement with the provided ground truth. The dramatic contrast in performance for the additional 19 test signals indicates that the classifiers were sharply tuned to the deterministic-like signals of the training set and not amenable to grossly deviant signals. This conclusion is further reinforced by interpreting the network outputs, as elaborated below.

Besides classification accuracy, memory requirements, training, and testing times are also of concern, particularly for real-time implementations. The AKC took the longest training time, while the RKC was quicker than LVQ but provided superior results. While determining the class of a test signal, the Pi-Sigma and RBF networks are about twice as fast than the other networks which exhibit comparable speeds. More accurate timing estimates could be obtained through a careful separation of CPU and input/output times.

\section{EVIDENCE INTEGRATION AND DECISION MAKING}

We observe that the DARPA Standard Data Set I is a simplified characterization of real-life short-duration oceanic signals, whose detection and classification is a much more difficult problem. Since different classification techniques have different inductive biases, a single method cannot give the best results for all signal types. Rather, more accurate and robust classification can obtained by combining the outputs (evidences) of multiple classifiers based on neural network and/or statistical pattern recognition techniques. With this motivation, we present two such techniques for evidence combination in this section.

It has been recently shown that training multilayer feedforward networks by minimizing the expected mean square error (MSE) at the outputs and using a $0 / 1$ teaching function yields network outputs that approximate posterior class probabilities [61]-[63]. In particular, the MSE is shown to be equivalent to

$$
\mathrm{MSE}=K_{1}+\sum_{c} \int_{x} D_{c}(x)\left(P(c / x)-f_{c}(x)\right)^{2} d x
$$

where $K_{1}$ and $D_{c}(x)$ depend on the class distributions only, $f_{c}(x)$ is the output of the node representing class $c$ given an input $x, P(c / x)$ denotes the posterior probability, and the summation is over all classes. Thus, minimizing the (expected) MSE corresponds to a weighted least squares fit of the network outputs to the posterior probabilities.

This result gives a sound mathematical basis for the interpretation of the network outputs, and for using an integrator to combine the outputs from multiple classifiers to yield a more accurate classification. For very low values of MSE, $f_{c}(x)$ approximates $P(c / x)$ according to (17). Let $f_{c, i}(x)$ be the output of the node that denotes membership in class $c$ in the $i$ th neural classifier. We expect that, for all $i, x$,

$$
\sum_{c} f_{c, i}=1
$$

Similarly, if the posteriori estimate is very good, one would expect for all $c, i$

$$
\frac{1}{N} \sum_{j=1}^{N} f_{c, i}\left(\boldsymbol{x}_{j}\right)=P(c)
$$


where $j$ indexes the $N$ training data samples, and $P(c)$ is obtained by counting the frequency of class $c$ in the training data.

Indeed, both (18) and (19) are observed to be quite accurate for almost all of the 179 test signals for both RKC and AKC, as well as for the PSN. For 16 of the 19 deviant signals, the summation of (18) is less than 0.5 strongly indicating that these signals do not resemble any signal in the training set.

Based on the interpretation of the outputs as a posterior class probabilities, two methods for evidence combination were proposed and used [52].

1) Entropy-Based Integrator: In this method, a weighted average of the outputs of $n$ different classifiers is first performed, with a larger entropy resulting in a smaller weight. The integrator then selects the class corresponding to the maximum value, providing this value is above a threshold. Otherwise, the input is considered as a false alarm, since there is no strong evidence that it belongs to any of the $n$ classes.

First, for every classifier, the entropy is calculated using normalized outputs $y_{c, i}^{\prime}=y_{c, i} / \sum_{c} y_{c, i}$. The weight given to each classifier (normalized) output differs from sample to sample according to the (approximated) entropy at the output of that classifier, as follows:

$$
H(c)=\frac{1}{n} \sum_{i=1}^{n} \frac{y_{c, i}}{-\sum_{c} y_{c, i}^{\prime} \ln y_{c, i}^{\prime}}
$$

assigned class label $=c: \max H(c)$.

In this way, the outputs of a classifier with several similar values get a lower weighting as opposed to classifiers that strongly hypothesize a particular class membership. Our initial experiment in combining the results of the RKC and the PSN yielded $100 \%$ accuracy among the 179 test signals and 8/19 (42\%) agreement with the ground truth provided for the deviant signals. More significantly, the values of $\max \{H(c)\}$ obtained for the set are significantly lower, thus indicating that this metric can be used to detect false alarms and unknown signals.

2) Heuristic Combination of Confidence Factors: This approach is inspired by techniques for parallel combination of rules in expert systems. Certainty factors were introduced in the MYCIN expert system for reasoning in expert systems under uncertainty, and reflect the confidence in a given rule [64]. The original method of rule combination in MYCIN was later expressed in a more probabilistic framework by Heckerman [65], and serves as the basis for the method proposed below.

First, the outputs, which are in the range $[0,1]$, are mapped into certainty or confidence factors (CF's) in the range $[-1,1]$ using a $\log$ transformation. Then, a MYCIN-type rule is used to combine the CF's for each class. The advantage of this combination rule is that it makes the result invariant to the sequence in which the different network outputs are combined.
The individual CF's are first obtained using

$$
\mathrm{CF}_{c, i}=\log _{n}\left((n-1 / n) y_{c, i}+1 / n\right)
$$

where subscript $i$ denotes the classifier as before. For each class $c$, as positive CF's and all negative CF's are combined separately. The resultant positive and negative CF's are combined in the final step, to obtain a combined confidence $\mathrm{CF}_{c}$, for each class $c$. The classification decision is:

$$
\text { assigned class label }=c: \max \mathrm{CF}_{c} \text {. }
$$

The equations used for combining the CF's are similar but not identical to those used in the original MYCIN [64]. For a given class, $c$, let the individual confidences obtained from two classifiers be $a$ and $b$, respectively. Then the confidence $C_{c}(a, b)$ obtained on combining these two values is given by

$$
\begin{aligned}
\mathrm{CF}_{c} & (a, b) \\
& =1-(1-a)(1-b) \quad \text { if } a>0 \quad \text { and } \quad b>0 \\
& =-\mathrm{CF}_{c}(-a,-b) \quad \text { if } a<0 \quad \text { and } \quad b<0 \\
& =a+b, \quad \text { otherwise. }
\end{aligned}
$$

An experiment in combining the results of the RKC and the PSN using confidence factors also yielded $100 \%$ accuracy among the 179 test signals and 9/19 (47\%) agreement with the ground truth provided for the deviant signals. Again, the values of $\max \mathrm{CF}_{c}$ were much lower for the deviant signals, yielding another metric for detecting false alarms and unknown signals. Indeed, by varying the threshold for the minimum acceptable value for max $H(c)$ or max $\mathrm{CF}_{c}$, one can obtain a range of classification accuracy versus false alarm rates, and be able to choose a suitable trade-off point.

\section{CONCLUding REMARKS}

Hybridization of algorithms is emerging as an important approach to solving problems. Each algorithm is a realization of one approach to a solution, and often a synergistic approach to the problem yields a better solution than making further improvements on a single approach. In fact, increasing the sophistication of a particular technique may not take us very far, as is witnessed by the history of LVQ. Rather, for difficult real-world problems like detection and classification of oceanic signals, it is crucial to have good preprocessing and feature selection techniques combined with efficient neural network classifiers and robust methods or integrated decision-making. Good performance is required at every stage, and cooperation is also desirable between stages. In our classifier, these principles are exemplified by the coupling of the selection feature vectors with the choice of neural classifiers, and by the use of evidence combination techniques in a situation when the capabilities of a single classifier are fundamentally limited.

\section{ACKNOWLEDGMENT}

The authors thank James Whiteley and Russell Still at Tracor Applied Sciences, Inc., and the University of Texas 
team of J. K. Aggarwal, Srinivasa Chakravarthy, Chen Chau Chu, Ed Powers, Irwin Sandberg, and Yoan Shin for contributions at various stages of the project.

\section{REFERENCES}

[1] L. Deuser and D. Middleton, "On the classification of underwater acoustic signals: An envinomentally adaptive approach," The Acoustic Society of America, vol. 65, pp. 438-443, 1979.

[2] C. H. Chen, "Automatic recognition of underwater transient signals-a review," in Proc. ICASSP, pp. 1270-1272, 1985.

[3] Special Issue, "Underwater Acoustic Signal Processing," IEEE J. Ocean. Eng., p. 2-278, Jan. 1987.

[4] R. J. Urick, Principles of Underwater Sound. New York: McGraw-Hill, 2nd Ed., 1975.

[5] W. Y. Huang and R. P. Lippmann, "Neural network and traditional classifiers," Neural Inform. Processing Syst., pp. 387-396, 1987.

[6] R. P. Lippmann, "Review of neural networks for speech recognition," Neural Computation, vol. 1, pp. 1-38, 1989.

[7] J. Ghosh and K. Hwang, "Mapping neural networks onto message-passing multicomputers," J. Parallel and Distributed Computing, vol. 6, pp. 291-330, Apr. 1989.

[8] K. Ng and R. P. Lippmann, "Practical characteristics of neural network and conventional pattern classifiers," in Advances in Neural Information Processing Systems - III, pp. 970-976, 1991.

[9] Y. L. Cun, J. S. Denker, and S. A. Solla, "Optimal brain damage," in Advances in Neural Information Processing Systems-II, pp. 598-605, 1990.

[10] S. Beck and J. Ghosh, "Noise sensitivity of static neural classifiers," in SPIE Conf. Sci Artificial Neural Networks SPIE Proc., vol. 1709, Apr. 1992.

[11] D. Lowe and A. R. Webb, "Optimized feature extraction and the bayes decision in feed-forward classifier networks," IEEE Trans. $P A M I$, vol. 13, pp. 355-364, Apr. 1991.

[12] J. Makhoul, "Pattern recognition properties of neural networks," in Proc. Ist IEEE Workshop on Neural Networks for Signal Processing, pp. 173-187, Sept. 1991.

[13] J. Denker et al. "Large automatic learning, rule extraction and generalization," Complex Systems, vol, 1, pp. 877-922, 1987.

[14] E. Levin, N. Tishby, and S. A. Solla, "A statistical approach to learning and generalization in layered neural networks," Proc. $I E E E$, vol. 78 , pp. $1568-74$, Oct. 1990.

[15] J. E. Moody, "Note on generalization, regularization and architecture selection in nonlinear learning systems," in IEEE Workshop Neural Networks for Signal Processing, pp. 1-10, 1991.

[16] P. J. Werbos, "Links between artificial neural networks and statistical pattern recognition," in I. K. Sethi and A. Jain, Eds., Artificial Neural Networks and Statistical Pattem Recognition. Amsterdam: Elsevier Science, 1991, pp. 11-32.

[17] S. Raudys and A. K. Jain, "Processing of textured images using neural networks," in I. K. Sethi and A. Jain, Ed. Antificial Neural Networks and Statistical Pattern Recognition. Amsterdam: Elsevier Science, 1991, pp. 33-50.

[18] J. Ghosh et al., "Adaptive kernel classifiers for short-duration oceanic signals," in IEEE Conf. Neural Networks for Ocean Engineering, pp. 41-48, Aug. 1991.

[19] J. Ghosh, L. Deuser, and S. Beck, "Impact of feature vector selection on static classification of acoustic transient signals," in Government Neural Network Applications Workshop, Aug. 1990.

[20] Y. Shin and J. Ghosh, "The pi-sigma network: An efficient higherorder network for pattern classification and function approximation," in Proc. Joint Conf. Neural Networks, pp. I: 13-18, July 1991.

[21] S. Beck, L. Deuser, R. Still, and J. Whiteley, "A hybrid neural network classifier of short duration acoustic signals," in Proc. IJCNN, pp. I:119-124, July 1991

[22] B. Widrow and S. D. Stearns, Adaptive Signal Processing. Englewood Cliffs, NJ: Prentice-Hall, 1985.

[23] R. P. Gorman and T. J. Sejnowski, "Analysis of hidden units in a layered network trained to classify sonar targets," Neural Networks, pp. 1:75-89, 1988.

[24] J. Specht, "Probabilistic neural networks," Neural Neworks, pp. $45-74,1990$.

[25] J. M. Combes, A. Grossman, and P. Tchamitchian, Eds., Wavelets: Time-Frequency Methods and Phase Space. New York: SpringerVerlag, 1989.
[26] Y. H. Pao, T. L. Hemminger, D. J. Adams, and S. Clary, "An episodal neural-net computing approach to the detection and interpretation of underwater acoustic transients," in Conf. Neural Networks for Ocean Eng., pp. 21-28, 1991.

[27] O. Rioul and M. Vetterli, "Wavelets and signal processing," IEEE Signal Processing Magazine, pp. 14-38, Oct. 1991.

[28] T. Lefebvre, J. M. Nicolas, and P. Degoul, "Numerical to symbolical conversion for acoustic signal classification using a two-stage neural architecture," in Proc. Int. Neural Network Conf., pp. 119-122, June 1990.

[29] S. Geman, E. Bienenstock, and R. Doursat, "Neural networks and the bias/variance dilemma," Neural Computation, vol. 4, pp. 1-58, 1992.

[30] T. D. Sanger, "Optimal unsupervised learning in a singe-layer linear feedforward neural network," Neural Networks, vol. 2, pp. $459-474,1989$

[31] T. Kohonen, Self-Organization and Associative Memory. Berlin: Springer-Verlag, 3rd ed., 1989.

[32] R. P. Lippmann, "Pattern classification using neural networks," IEEE Commun. Mag., pp. 47-64, Nov. 1989.

[33] K. Ng and R. P. Lippmann, "A comparative study of the practical characteristics of neural network and conventional pattern classifiers," Advances in Neural Information Processing Systems-III, pp. $970-975,1990$

[34] D. S. Broomhead and D. Lowe, "Multivariable functional interpolation and adaptive networks," Complex Systems, vol. 2, pp. 321-355, 1988.

[35] J. Moody and C. J. Darken, "Fast learning in networks of locallytuned processing units," Neural Computation, vol. 1, pp. 281-294, 1989.

[36] P. K. Simpson, "Fuzzy min-max classification with neural networks," in Proc. IEEE Conf. Neural Networks for Ocean Eng., pp. 291-300, Aug. 1991

[37] T. Kohonen, G. Barna, and R. Chrisley, "Statistical pattern recognition with neural networks: Benchmarking studies," in IEEE Annual Int. Conf. Neural Networks, July 1988.

[38] S. M. Weiss and C. A. Kulikowski, Computer Systems That Learn, San Mateo, CA: Morgan Kaufmann, 1991.

[39] M. I. Jordan, "Serial order: A parallel, distributed processing approach," in J. L. Elman and D. E. Rumelhart, Eds., Advances in Connectionist Theory: Speech. Hillsdale: Lawrence Erlbaum, 1989.

[40] J. L. Elman, "Finding structure in time," Cognitive Science, vol. 14, pp. $179-211,1990$

[41] R. Hecht-Nielsen, Neurocomputing. Reading, MA: AddisonWesley, 1990.

[42] M. Sato, "A real time learning algorithm for recurrent analog neural networks," Bio. Cybern., vol. 62, pp. 237-241, 1990.

[43] A. G. Ivakhnenko, "Polynomial theory of complex systems," IEEE Trans. Syst. Man, Cybern., vol. 1, pp. 364-378, Oct. 1971.

[44] Y.-H. Pao, Adaptive Pattern Recognition and Neural Networks. Reading, MA: Addison-Wesley, 1989

[45] J. Kowalski, E. Hartman, and J. Keeler, "Layered neural networks with Gaussian hidden units as universal approximators," Neural Computation, vol. 2, pp. 210-215, 1990.

[46] M. Niranjan and F. Fallside, "Neural networks and radial basis functions in classifying static speech patterns," Tech. Rep. CUED/ FINFENG / TR22, 1988

[47] D. deSieno, "Adding conscience to competitive learning," in IEEE Annual Int. Conf. Neural Networks, pp. 1117-1124, 1988.

[48] C. L. Giles and T. Maxwell, "Learning, invariance, and generalizaiton in a high-order neural network. Applied Optics, vol. 26, pp. $4972-4978,1987$.

[49] M. R. Lynch and P. J. Rayner, "The properties and implementation of the non-linear vector space connectionist model," in Proc. First IEE Int. Conf. Artificial Neural Networks, pp. 184-190, Oct. 1989.

[50] J. Ghosh and Y. Shin, "Efficient higher-order networks for function approximation and classification," IEEE Trans. Neural Networks, 1992

[51] K. Hornik, M. Stinchcombe, and H. White, "Multilayer feedforward networks are universal approximators," Neural Networks, vol. 2, pp. 359-366, 1989.

[52] J. Ghosh, S. Beck, and C. C. Chu, "Evidence combination techniques for robust classification of short-duration oceanic signals," in SPIE Conf. Adaptice Learning Systems, SPIE Proc., vol. 1706, Apr. 1992. 
[53] S. Geva and J. Sitte. "Adaptive nearest neighbor classification," IEEE Trans. Neural Networks, vol. 2, pp. 318-322, 1991.

[54] S. Lee and R. M. Kil, "Multilayer feedforward potential function network," In Proc. Second Int. Conf. Neural Networks, pp. 161-171, 1988.

[55] T. Poggio and F. Girosi, "Networks for approximation and learning." Proc. IEEE, vol. 78. pp. 1481-1497, Sept. 1990.

[56] J. Park and I. W. Sandberg, "Universal approximation using radial basis function networks," Neural Computation, vol. 3, pp. 246-257, 1991.

[57] R. Hecht-Neilsen, "Counterpropagation networks," Appl. Optics, vol. 26, pp. 4979-4984, 1987.

[58] S. Chakravarthy, J. Ghosh, L. Deuser, and S. Beck, "Efficient training procedures for adaptive kernel classifiers," in Neural Nerworks for Signal Processing, pp. 21-29, IEEE Press, 1991.

[59] R. O. Duda and P. E. Hart, Pattern Classification and Scene Analysis. New York: Wiley, 1973.

[60] K. Fukunaga, Introduction to Statistical Pattem Recognition, 2nd Ed. New York: Academic, 1990.

[61] M. D. Richard and R. P. Lippmann, "Neural network classifiers estimate bayesian a posteriori probabilities," Neural Computation, vol, 3. pp. 461-483, 1991

[62] H. Gish, "A probablistic approach to the understanding and training of neural network classifiers," in Proc. Int. Conf. ASSP, pp. 1361-1364. Apr. 1990.

[63] P. A. Shoemaker, M. J. Carlin, R. L. Shimabukuro, and C. E. Priebe. "Least squares learning and approximation of posterior probabilities on classification problems by neural network models," in Proc. 2nd Workshop Neural Networks, WNN-AIND9l, pp 187-196, Feb. 1991

[64] E. H. Shortcliffe and B. G. Buchanan, "A model of inexact reasoning in medicine." Mathematical Biosciences, vol. 23, pp. $351-379,1975$

[65] D. Heckerman, "Probabilistic interpretation for MYCIN's uncertainty factors," in L.N. Kanal and J. F. L.emmer, Eds., Uncertainty in Artificial Intelligence. North-Holland, 1986, pp. 167-196.

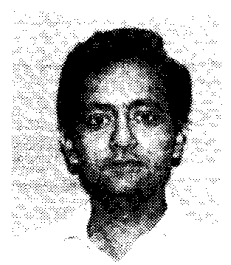

Joydeep Ghosh received the B.Tech. degree in electrical engineering from IIT, Kanpur. India in 1983, and the M.S. and $\mathrm{Ph}$ D. degrees in computer engineering from the University of Southern California, in 1985 and 1988, respectively. He was the first member of the School of Engineering at USC to be awarded an "AllUniversity Predoctoral Teaching Fellowship" for four years. His doctoral dissertation dealt with the architecture and applications of massively parallel systems such as artificial neural net-

works.

He is currently an Assistant Professor in the Department of Electrical and Computer Engineering at the University of Texas, Austin. His areas of interest are artificial neural systems and their applications in image analyses and signal processing, computer architecture, and massively parallel processing. He has more than 40 referecd publications in these areas. He served as the general chairman for the SPIE/SPSE Conference on Image Processing Architectures, Santa Clara, Feb. 1990, and is a member of the editorial board of IEEE Computer Society Press and of Pattern Recognition. Dr. Ghosh is the recepient of the 1992 Darlington Award for the Best Paper in IEEE Trans. Circuits and Systems.

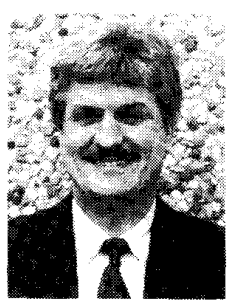

Larry M. Deuser received the BSEE and MSEE degrees from Purdue University in 1962 and 1964, respectively, and the Ph.D. from the University of Texas at Austin in 1975

From 1968 to 1977 he was with the Applied Research Laboratories of the University of Texas, Austin. TX. His work involved signal and image processing for both hydrospace and aerospace remote sensing. He was with Hughes, Culver City, CA from 1964 to 1967 working on early synchronous satellite and synthetic aperture radar analysis. He has authored or co-authored more than 80 papers and reports. He is the Director of the Signal and Image Processing Department at Tracor Applied Sciences, Inc. Austin, TX. Since joining Tracor in 1977, he has performed and directed efforts in innovative approaches to signal and image processing for automatic and semiautomatic detection/classification. The techniques involved include artificial neural networks, parametric and nonparametric classification, optimal feature extraction and selection, and adaptive processing. The applications have been to the underwater acoustical and optical domains.

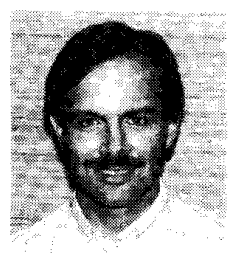

Steven I. Beck received the BSEE and the MEE degrees in clectrical engineering from Rice University in 1981 and 1982, respectively.

From 1982 to 1984 he worked with Ford Aerospace in Palo Alto, CA, developing automatic detection and classification systems for use in advanced digital communications. Since 1985, he has been with Tracor Applied Sciences, Inc., Austin, TX, where he is currently a Principal Scientist on several underwater acoustics projects involving nontraditional signal processing and neural networks. His professional interests include adaptive signal and image processing, neural networks, and real-time information processing systems 